\title{
Velocidad. Significados manifestados por estudiantes universitarios a partir de representaciones gráficas
}

\author{
Abraham Cuesta Borges, Universidad Veracruzana (México) \\ Juana Elisa Escalante Vega, Universidad Veracruzana (México) \\ Juan Francisco Ruiz Hidalgo, Universidad de Granada (España)
}

Recibido el 2 de Mayo de 2015; aceptado el 1 de octubre de 2015

\begin{abstract}
Velocidad. Significados manifestados por estudiantes universitarios a partir de representaciones gráficas

\section{Resumen}

En el contexto de un curso nivelatorio de Cálculo para estudiantes que inician la Licenciatura de Economía, se les propusieron tareas de interpretación y construcción de gráficos funcionales con objeto de determinar cómo entienden la relación lineal que se establece entre las variables velocidad, distancia y tiempo. Mediante un análisis de contenido de las respuestas de los estudiantes, se obtienen confusiones y errores detectados en investigaciones previas $y$, además, se consigue identificar significados que los estudiantes atribuyen a cada una de las variables. A pesar de que los estudiantes manifiestan su conocimiento de la relación algebraica de dichas variables, se observan diversas interpretaciones que manifiestan que el significado que asignan a velocidad es incompleto.
\end{abstract}

Palabras clave. Función; Velocidad; Interpretaciones; Errores; Significado. graficas.

Velocidade. Significados expressos por estudantes universitários desde representações

\section{Resumo}

No contexto de um curso de nivelamento de Cálculo para alunos iniciantes em Bacharelado em Economia, propusemos tarefas de interpretação e construção de gráficos funcionais, a fim de determinar como eles entendem a relação linear estabelecida entre velocidade, distância e tempo. Por meio de uma análise de conteúdo, detectamos confusões e erros já referidos em pesquisas anteriores. Além disso, identificamos significados que os alunos atribuem a cada variável. Embora eles expressem conhecimento da relação algébrica dessas variáveis, observamos diversas interpretações indicando que osignificado atribuído a velocidade está incompleto.

Palavras chave. Função; Velocidade; Interpretações; Erros; Significado.

Speed. Meanings expressed by college students from graphical representation.

\section{Abstract}

In the context of a Calculus levelling course for students beginning the Degree of Economics, we proposed tasks of interpretation and construction of functional graphs, in order to determine how they

Para citar: Cuesta, A., Escalante, J.E., Ruíz-Hidalgo, J.F. (2016). Velocidad. Significados manifestados por estudiantes universitarios a partir de representaciones gráficas. Avances de Investigación en Educación Matemática, 9, 105 - 125.

(C) Sociedad Española de Investigación en Educación Matemática (SEIEM). www.seiem.es 
understand the linear relationship established among speed, distance and time. By means of a content analysis, we detect confusions and errors already referred in previous research, as well as we identify meanings that students attribute to each variable. Although students express their knowledge of algebraic relationship of these variables, we observe several interpretations that suggest the meanings assigned to speed is incomplete.

Key words. Function: Speed; Interpretations; Errors; Meaning.

Vitesse. Significations exprimées par les étudiants à partir de graphiques.

\section{Résumé}

Dans le cadre d'un cours de nivellement de Calcul pour les étudiants commençant les études d'économie, nous avons proposé des tâches d'interprétation et la construction de graphes fonctionnels afin d'étudier leur compréhension de la relation linéaire entre la vitesse, la distance et le temps. Au moyen d'une analyse de contenu, nous détectons les confusions et les erreurs trouvées dans les recherches précédentes. En outre, nous identifions les significations que les élèves attribuent à chaque variable. Bien que les étudiants expriment leur connaissance de la relation algébrique de ces variables, on observe plusieurs interprétations affirmant que les significations attribuées à la vitesse sont incomplètes.

Paroles clés. Fonction; Vitesse; Interprétations; Erreurs; Signification.

\section{Introducción}

En algunas universidades mexicanas se ha detectado que los resultados en las asignaturas de Matemáticas de los estudiantes que cursan la Licenciatura de Económicas son alarmantemente bajos. En el caso de la Universidad Veracruzana, incluso, desde los resultados del examen de ingreso se exhibe la existencia de dificultades derivadas del hecho de que el estudiante no ha desarrollado la capacidad de utilizar el concepto matemático de una manera adecuada en situaciones contextualizadas, aun cuando éstas formen parte de sus experiencias personales y conocimientos anteriores.

Una de las soluciones propuestas, que se ha puesto en marcha en diferentes universidades, es la creación de un curso cero o curso nivelatorio de matemáticas. En la Licenciatura de Económicas de la Universidad Veracruzana se lleva realizando este curso desde el año 2000, con el objeto de proporcionar a los estudiantes de nuevo acceso la opción de consolidar o, en algunos casos, adquirir habilidades elementales de cálculo numérico-algebraico, destrezas y aptitudes para la representación gráfica de relaciones cuantitativas, e iniciar la aplicación de una evaluación de tipo diagnóstica para orientar el trabajo de los profesores. Uno de los focos más importantes de este curso opcional es el trabajo con funciones y, más concretamente, con su representación gráfica, que influye en la adquisición de conceptos del análisis que se relacionan de manera directa en la comprensión de la teoría económica.

En este contexto, y para fines de investigación, el equipo docente decide ajustar la evaluación diagnóstica con el objeto de profundizar más en las dificultades con las que acceden los estudiantes en la lectura e interpretación de gráficas. De este modo, se preparan cinco cuestiones sobre funciones, especialmente centradas en su representación gráfica e interpretación de elementos globales. El objetivo de este trabajo es describir los resultados de esta evaluación, para precisar los significados que los estudiantes egresados de Bachillerato manifiestan sobre cada una de las variables que aparecen en las tareas propuestas. Más concretamente, se centra la atención del 
estudiante en el concepto de velocidad, trabajado en diferentes cursos de educación secundaria. La intención es determinar si el estudiante logra interpretar y construir representaciones gráficas en situaciones contextualizadas mediante la identificación de características globales de las mismas.

El estudio identifica dificultades relacionadas con dos confusiones recogidas en la bibliografía (Janvier, 1987): la confusión gráfico-dibujo y la confusión verbal-gráfica. Se pudo constatar, además, que los estudiantes no reconocen adecuadamente la dependencia funcional entre las variables implicadas en una gráfica, unido a la tendencia a asumir linealidad y a la insuficiente comprensión del significado de pendiente. Estas dificultades son producidas (Deulofeu, 1993, 1995; Leinhardt, Zaslavsky \& Stein, 1990) por el efecto combinado de los significados que poseen los estudiantes sobre el concepto y del conocimiento que tienen sobre el contexto en que se deben realizar dichas tareas.

Además de estas dificultades, detectamos la ausencia de significado que manifiestan los estudiantes de la noción de velocidad, concretados en tres problemas de sentido: ausencia de significado de la pendiente de la recta, ausencia de significado de la relación entre las variables y dotar de sentido funcional a situaciones icónicas. Se pudo corroborar que, en efecto, el significado de función y de relación entre variables que expresan los estudiantes parece ser insuficiente para acceder a las complejas estructuras y relaciones que exige el estudio de las funciones, y en particular sus distintos sistemas de representación como el lenguaje gráfico.

El trabajo comienza con una descripción del marco teórico en el que se basa y se acompaña de los antecedentes que consideramos más influyentes para el estudio. La siguiente sección es metodológica y en ella se describen los participantes, el instrumento y el método de análisis de respuestas. En los resultados se analizan las cuestiones por separado y se destaca, en cada una de ellas, los sentidos más relevantes detectados. Para terminar, se presentan las conclusiones.

\section{Marco teórico y antecedentes}

Aportamos en este apartado los pilares teóricos que lo fundamentan, así como las referencias previas que determinan las dificultades que los estudiantes manifiestan sobre el concepto de función y sus representaciones.

\subsection{Sistemas de representación}

El término sistemas de representación es complejo, en tanto que enclaustra diversos significados y puede ser utilizado en una gran variedad de ámbitos; sin embargo, su exhaustiva clarificación (Janvier, 1987; Rico 2009), en educación matemática, permite concebirlo como parte esencial del aparato conceptual necesario para analizar los procesos de aprendizaje y comprensión de las matemáticas. De acuerdo con Castro y Castro (1997), las representaciones constituyen el conjunto de "notaciones simbólicas o gráficas, mediante las que se expresan conceptos y procedimientos matemáticos, así como sus características y propiedades más relevantes" (Castro \& Castro 1997, p. 96), que se clasifican en registros de representación (Duval, 1999) y de cuya comprensión depende de si la representación mental es parte de una red de representaciones (Hitt, 2001; Martí, \& Pozo, 2000; Rico, 2009). 
Las investigaciones actuales, que centran su atención en el estudio del proceso cognitivo, ponen especial énfasis en el estudio de las diferentes representaciones, físicas o mentales, que son imprescindibles y dan significado a los conceptos matemáticos estudiados. Desde Davis, Young y McLoughlin (1982), Janvier (1987), Duval (1988), Goldin (1998), la idea de sistema de representación (Castro, \& Castro 1997; Hitt, 2001; Rico, 2009), es un campo de estudio en educación matemática. En este sentido, un objeto especial de análisis ha sido la instrucción sobre el concepto de función, en cuyo proceso de aprendizaje los alumnos se pueden encontrar con varias representaciones (gráficas, expresiones algebraicas, tablas, diagramas de flechas, etc.)

\subsection{Tipos de tareas de representación gráfica de funciones y errores}

En el estudio de la representación gráfica de funciones, existen dos tareas que son esenciales, a saber: la interpretación y la construcción. La interpretación (global o local) se refiere al acto de adquirir sentido de una situación, por medio de una gráfica, una ecuación o una tabla; en ella se obtiene un significado (o información) (Leinhardt et al, 1990). En la construcción, en cambio, el alumno genera una cosa nueva, consiste en pasar de la descripción verbal de una situación a la gráfica y/o tabla; aunque toda construcción se apoya a menudo en algún tipo de interpretación. Janvier (1987) examina la representación del concepto de función a través de dos tipos fundamentales de traducciones (conversiones en términos de Duval, 1999): (i) la interpretación, que consiste en pasar, por ejemplo, de la gráfica de una situación a su descripción verbal y (ii) la construcción, que consiste en pasar de la descripción verbal de una situación a la gráfica y/o tabla.

Para Janvier (1987) los errores se deben a confusiones (contaminaciones) entre las diferentes representaciones y sitúa como las más significativas las confusiones visuales: la confusión gráfico-dibujo, la confusión verbal-gráfica y la confusión intervalo-punto. Aunque, también considera las confusiones provocadas por la experiencia personal (personal distractors) que pueden surgir, de manera simultánea con las confusiones visuales, y provocar mayor dificultad en las tareas de interpretación y construcción de gráficas. Mientras que, en Leinhardt et al. (1990) se consideran cuatro tipos de dificultades, a saber: la confusión entre la pendiente y la altura, la confusión entre un intervalo y un punto, la consideración de una gráfica como un dibujo y la consideración de una gráfica como construida por un conjunto discreto de puntos.

Las dificultades en estas dos tareas (interpretación y construcción) tienen incidencia en el proceso de aprendizaje del propio concepto de función, al extremo de que pueden convertirse en un obstáculo para el alumno. De hecho, así se constata en investigaciones recientes sobre tareas de interpretación y construcción de gráficos. Bagni (2004), por ejemplo, destaca la influencia de la visualización en la representación gráfica y los obstáculos en la identificación total de una función a su gráfica cartesiana, mientras que Hitt (2001) pone de manifiesto la dificultad para conectar el aspecto figural de una gráfica con el aspecto analítico de un fenómeno.

Fabra y Deulofeu (2000) realizan un análisis de respuestas de un grupo de alumnos a preguntas relacionadas con situaciones gráficas descontextualizadas. Concluyen cómo en las percepciones del alumno predominan determinados prototipos; de acuerdo con los autores algunas de las gráficas se reducen al trazado de rectas o segmentos de recta, parábolas o hipérbolas, que son en esencia gráficos de modelos de funciones 
conocidas (estudiadas) por el alumno. Más recientemente, Ortega y Pecharromán (2014) presentan algunos errores que manifiestan los alumnos al trabajar con funciones a través de sus gráficas, y realizan una clasificación de los errores que surgen en la medida que los alumnos van adquiriendo los conceptos en diferentes estadios de aprendizaje (semiótico, estructural y autónomo).

Los trabajos de Dolores y colaboradores (Dolores, Bello \& Millán, 2002; Dolores, 2004; Dolores, Chi, Canul, Cantú \& Pastor, 2009) destacan concepciones de estudiantes de secundaria y bachillerato acerca de elementos de las gráficas de funciones desde un punto de vista semiótico. La rapidez es uno de los tópicos por los que se pregunta (Dolores et al., 2009). La mayoría de los estudiantes dan representaciones gráficas de la rapidez asociándola con su magnitud y no con la pendiente o cociente de magnitudes. En otro trabajo (Dolores et al., 2002) el tópico es la velocidad y su objetivo es detectar concepciones alternativas sobre velocidad media, velocidad instantánea y trayectoria de cuerpos en movimiento que se desprenden de la lectura de gráficas cartesianas de coordenadas tiempo-distancia. El estudio se aplicó a estudiantes de secundaria, bachillerato y universidad y a profesores de preparatoria y secundaria. Entre las concepciones detectadas destacamos las que relacionan mayor velocidad con mayor velocidad media y trayectoria del objeto con la representación gráfica de la velocidad. Centrado específicamente en velocidad, Dolores et al. (2009) analizan los tipos de representaciones gráficas que usan los estudiantes de secundaria y bachillerato para representar la velocidad y la rapidez. Encuentran cinco tipos: rectas, columnas, puntos, pictóricas y curvas y concluyen que la mayoría de los estudiantes dan representaciones gráficas de la rapidez asociándola con su magnitud y no con la pendiente o cociente de magnitudes de los cambios.

Recientemente, han aparecido un conjunto de publicaciones relacionadas con el término velocidad en educación matemática enfocadas fundamentalmente a detectar estrategias de resolución de problemas verbales de velocidad y realizar comparaciones internacionales (Jiang, 2009, 2013; Jiang \& Chua, 2010; Jiang, Hwang \& Cai, 2014), o más relacionadas con aspectos cognitivos como los trabajos de Zhou y colaboradores (Zhou, Peverly, Boehm \& Lin, 2000; Zhou, Peverly \& Lin, 2004).

En el caso particular de la ciencia económica, un aspecto importante para la comprensión es que las intuiciones del estudiante estén basadas en el conocimiento del estudiante sobre situaciones condicionadas por el hecho económico. Su núcleo teórico se halla en estrecha relación con conceptos, expresiones y modelos matemáticos, que implica la necesidad de una comprensión real y significativa de las matemáticas. Un estudio (Cuesta, 2007) fue realizado en el propio contexto de este trabajo, con el objeto de analizar las dificultades en el proceso de aprendizaje de los conceptos de función y extremo de una función. Puso de manifiesto que algunos estudiantes analizan una situación contextualizada atendiendo sólo a la lectura literal de las magnitudes involucradas (por ejemplo: el precio y el peso de bolsas de azúcar) sin establecer la relación manifiesta entre ellas.

\subsection{Significado de un concepto matemático escolar}

Además de la representación y de los errores que se cometen con las mismas, entendemos que en el significado de la noción de función incluye una perspectiva semántica. Entendemos el significado, de cualquier concepto matemático, desde la perspectiva del triángulo semántico propuesto por Frege (2013) compuesto por el 
signo con el que se expresa el concepto, su referencia y el sentido que se le atribuye cuando se usa. En matemática escolar, Rico (2012) identifica estas tres componentes con:

- Signo con sistemas de representación, o conjunto de símbolos y reglas con las que se representa el concepto.

- Referencia con estructura conceptual, la cual engloba las propiedades del concepto, los argumentos y proposiciones derivadas y los criterios de veracidad.

- Sentido con fenomenología, contextos, situaciones o problemas que dan sentido al concepto.

Mediante las tres componentes del triángulo semántico descritas interpretamos cómo los estudiantes entienden y comunican su significado de "velocidad" y lo relacionamos con los errores que cometen y que están caracterizados en la bibliografía.

\section{Metodología}

Este trabajo es un estudio descriptivo que se sirve de un cuestionario y de entrevistas individuales. Todas las cuestiones, formuladas verbalmente, giran en torno a un objeto gráfico sobre el cuál se realizan las preguntas. Las entrevistas individuales sirvieron para aclarar dudas de los investigadores acerca de la forma de resolución ejecutada por los estudiantes.

El análisis de las respuestas se realizó utilizando el método de análisis de contenido, entendido como el análisis riguroso, el examen y verificación de los contenidos en datos escritos, y cuya finalidad es descubrir la estructura interna de la comunicación, estudiando para ello su contenido semántico (Cohen, Manion \& Morrison, 2011; Rico \& Fernández-Cano, 2013).

\subsection{Participantes}

A los estudiantes que se inscriben en el primer curso de Licenciatura en Economía de la Universidad Veracruzana, se les ofrece la oportunidad de asistir al curso opcional introductorio previo al estudio del Cálculo I. Este curso, "Introducción a los Métodos Cuantitativos", tiene por objetivo proporcionar a los estudiantes de nuevo ingreso la opción de consolidar o, en algunos casos, adquirir habilidades elementales de cálculo numérico-algebraico, destrezas y aptitudes para la representación gráfica de relaciones cuantitativas, así como capacidad de razonamiento abstracto para el planteamiento y resolución de problemas matemáticos.

De los 110 alumnos matriculados durante el año académico 2013-2014, los 64 estudiantes que seleccionaron voluntariamente dicho curso introductorio conforman la muestra de este estudio. Cabe mencionar que todos los estudiantes han cumplido los requisitos de acceso a los estudios universitarios y al momento de aplicar la prueba de este estudio no han recibido ningún tipo de instrucción dentro de la universidad.

\subsection{Instrumento}

Se diseñó una prueba escrita, compuesta por problemas sobre situaciones contextualizadas en las que se requiere ejecutar tareas de interpretación y/o 
construcción de graficas (Leinhardt et al, 1990). Dichas situaciones fueron tomadas, con ligeras adaptaciones de contexto, de los trabajos de Azcárate y Deulofeu (1990) y de Shell Centre for Mathematical Education (1990).

El cuestionario está compuesto de cinco preguntas, de las cuales se analizan tres por motivos organizativos; todas ellas se basan en las relaciones entre diferentes sistemas de representación de funciones, principalmente de situaciones gráficas y enunciados verbales. Todas las tareas profundizan en la relación espacio recorrido y tiempo empleado, y para su solución se requiere identificar características que no se reconocen de manera inmediata, sino mediante un análisis del comportamiento

La primera tarea analizada es de tipo interpretativo, en ella se presenta una figura de la que se pide que se interprete mediante la respuesta a preguntas concretas. La segunda situación analizada es de tipo interpretativo/constructivo, en la que partiendo de un enunciado verbal se debe responder a algunas cuestiones para, finalmente, construir una gráfica que de sentido a la situación. La tercera situación analizada es de tipo constructivo, en la que se solicita, a partir de un enunciado verbal, la construcción de una tabla de valores y una gráfica que de sentido a la situación. Puesto que la cuarta y la quinta situaciones también son de tipo constructivo, similares a la tres, se decidió no considerarlas para este estudio.

De este modo, la investigación posee tres momentos:

1. Aplicación de prueba escrita: Se propone por un tiempo de 2 horas, durante la primera semana del curso introductorio, bajo la siguiente orientación: (i) no borrar ningún procedimiento escrito y (ii) en caso de no responder, se escriban las causas. Para mantener el anonimato de las respuestas, cada estudiante se clasifica de acuerdo al orden en que son registrados sus datos: $\mathrm{E}_{1}$ a $\mathrm{E}_{64}$.

2. Categorización de las actuaciones: El análisis de contenido de las respuestas escritas permitió elaborar una matriz que relaciona a cada estudiante con las respuestas a cada una de las preguntas. Se verificó que, ciertamente, se ponen de manifiesto dificultades e inconsistencias de naturaleza diferente, muchas de ellas vinculadas al nivel de conocimiento que se tiene sobre el concepto analizado (velocidad). Dicha clasificación será tratada, de manera particular, en el análisis de cada una de las situaciones propuestas.

3. Entrevista individual: Se seleccionaron 9 estudiantes, bajo el criterio de que las respuestas fueran representativas de la categorización realizada. Todas las entrevistas se grabaron en audio, en un contexto de intercambio de ideas, con la finalidad obtener datos sobre la forma en que los estudiantes llegan a la solución de las tareas planteadas. Con su análisis se intenta realizar una descripción interpretativa de las respuestas.

\subsection{Categorías}

Las categorías de análisis surgen tras realizar el análisis de contenido y tienen una doble vertiente. Por un lado se determinan los errores ya tipificados en investigaciones anteriores tales como: la confusión gráfico-dibujo, la confusión verbal-gráfica, la confusión entre la pendiente y la altura, la confusión entre un intervalo y un punto y la consideración de una gráfica construida por un conjunto discreto de puntos. Pero más allá de corroborar los hallazgos de investigaciones anteriores (Dolores et al., 2002; Fabra \& Deulofeu, 2000; Janvier, 1987; Leinhardt et al., 1990), intentamos explicarlos 
en términos de la interpretación y sentido personal del estudiante, es decir, a partir de la lectura que realiza de las nociones y/o variables implicadas en cada situación.

\section{Resultados}

A continuación se muestra el análisis de tres de las situaciones propuestas, comenzando por una clasificación de las actuaciones (correctas y erróneas). Se categorizan las incorrectas de acuerdo a la manifestación de alguna de las confusiones, y finalmente se describen los sentidos que posee el estudiante, a partir de la lectura que realiza de las variables implicadas. Este proceso se complementa con una descripción de lo hallado en las entrevistas.

\subsection{Situación 1 (tarea de interpretación)}

Para su solución se requiere cierto nivel de interpretación de las variables que intervienen, de manera explícita o implícita, así como de las relaciones descritas gráficamente. Se requiere, además, leer e interpretar la velocidad como pendiente de la recta. Las respuestas esperadas (sin considerar las explicaciones) son: (a) Mayor; (b) Mayor.

La figura muestra las gráficas "distancia contra tiempo" resultantes de la observación del movimiento de dos coches, denotados A y B, respectivamente. Se solicita responder las siguientes preguntas:

(a) ¿En el instante de tiempo $t=3$, la velocidad del coche A es menor, igual o mayor que la velocidad del coche B. Explica.

(b) ¿En el instante de tiempo $t=6$, la velocidad del coche A es menor, igual o mayor que la velocidad del coche B. Explica.

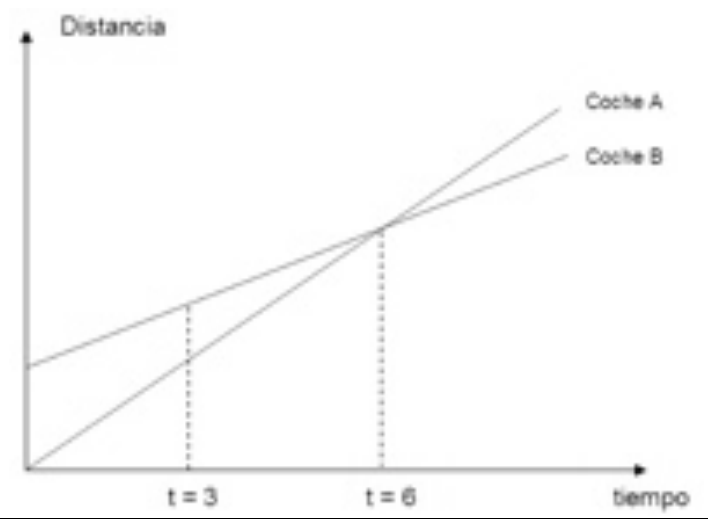

Figura 1. Enunciado de la Situación 1

El 19\% de los estudiantes (Tabla 1) responde de manera correcta al apartado a), mientras que al apartado b) el 6\%. En ambos casos, la explicación de que el coche A posee mayor velocidad que el $\mathrm{B}$, se basa en dos ideas: (i) el coche A recorre mayor distancia que el coche $\mathrm{B}$, en el mismo intervalo de tiempo, y (ii) la pendiente de la recta que representa al movimiento del coche A es mayor que la del coche $\mathrm{B}$.

Las respuestas del resto de los estudiantes, a ambos apartados, son erróneas (81\% y $94 \%$, respectivamente). A causa de dos confusiones manifiestas: pendiente-altura y 
gráfica-dibujo, que, en ocasiones combinadas, surgen de la lectura de los conceptos: distancia y velocidad, así como de la interpretación de la recta como recorrido físico, es decir la asociación de la recta a su imagen visual. De este modo, las respuestas pueden surgir de una combinación de los diferentes sentidos que el estudiante atribuye a partir de su lectura personal.

Tabla 1. Porcentaje de actuaciones, confusiones y sentidos en la situación 1

\begin{tabular}{llcc}
\hline \multirow{2}{*}{ Actuaciones } & Respuestas Correctas & Apartado a) & Apartado b) \\
\cline { 2 - 4 } & Respuestas Incorrectas & 81 & 6 \\
\hline $\begin{array}{l}\text { Confusiones } \\
\text { identificadas en la } \\
\text { literatura previa }\end{array}$ & Confusión pendiente-altura & 67 & 35 \\
\hline \multirow{2}{*}{$\begin{array}{l}\text { Sentidos asignados a las } \\
\text { variables }\end{array}$} & $\begin{array}{l}\text { Identifica altura como distancia } \\
\text { velocidad }\end{array}$ & 43 & 48 \\
\cline { 2 - 4 } & $\begin{array}{l}\text { Interpreta la recta como } \\
\text { recorrido físico }\end{array}$ & 40 & 38 \\
\cline { 2 - 4 } & Otras & 3 & 24 \\
\hline
\end{tabular}

Identifica altura como distancia: existe el reconocimiento de que la velocidad se debe hallar en términos de las variables distancia y tiempo; pero se realiza una lectura de la altura del eje vertical, en el sentido de que es interpretada como la distancia recorrida por el coche. La respuesta de E36 (Figura 2) ejemplifica la manera en que los estudiantes interpretan la distancia en el eje vertical.

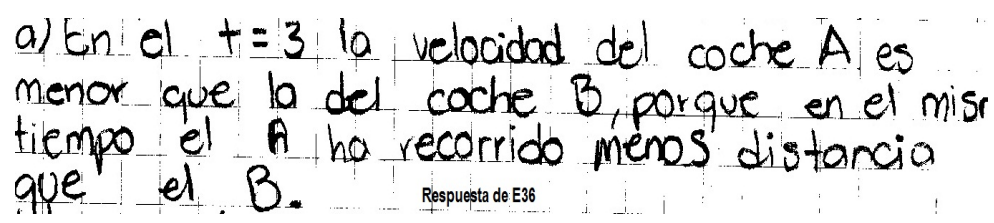

Figura 2. Respuesta del estudiante $\mathrm{E}_{36}$ en la situación 1

La interpretación de la altura como distancia, manifiesta en el $43 \%$ de las actuaciones, se puede apreciar en este fragmento de entrevista a $E_{36}$.

Profesor ¿Qué respondes a la primera pregunta?

$\mathrm{E}_{36} \quad$ Yo escribi que la velocidad de A es menor, porque el coche B ya lleva cierta distancia y el coche A comienza en cero.

Profesor ¿Cómo puedo observar que en $t=3$ la distancia del coche A es menor?

$\mathrm{E}_{36} \quad$ Es que la línea del coche A está por debajo que la línea del coche B.

Profesor ¿Y en el instante de tiempo $t=6$ ?

$\mathrm{E}_{36} \quad$ La velocidad e igual, el coche A recorre la misma distancia en el mismo tiempo, entonces lleva la misma velocidad. 
Identifica distancia como velocidad: no se reconoce la velocidad como una relación distancia - tiempo; por el contrario, se asocia sólo a la distancia, que es medida desde el eje horizontal hasta la recta (Figura 3).

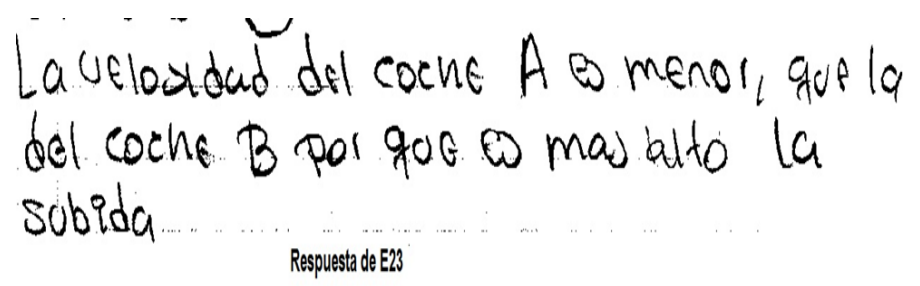

Figura 3. Respuesta del estudiante $\mathrm{E}_{23}$ en la situación 1

Este estudiante, durante la entrevista, se expresa en términos de que "el coche A no logra alcanzar a $\mathrm{B}$ en $\mathrm{t}=3$ ".

Profesor ¿Qué respondes a la primera pregunta?

$\mathrm{E}_{23} \quad$ Yo escribi que es menor la velocidad del coche A porque el coche B tiene una velocidad constante, $y$ el coche A apenas inicia.....

Profesor ¿Observas que en $t=3$ la velocidad de A es menor que la velocidad de B?

$\mathrm{E}_{23} \quad$ Si claro, no lo alcanza, lo llega a alcanzar un poco antes de $t=6$.

Profesor ¿Cuál tiene mayor velocidad en $t=6$ ?

$\mathrm{E}_{23} \quad$ El coche B no cambia mucho su velocidad, es casi constante, y el coche A inicia con una velocidad mínima pero va aumentando y alcanza a $B$.

Profesor Entonces, en $t=6$, ¿cuál tiene mayor velocidad?

$\mathrm{E}_{23} \quad$ Es igual porque se encuentran, la velocidad de B no cambia mucho y el coche A lo encuentra en la misma distancia.

Interpreta la recta como recorrido físico: se realiza una lectura de las rectas, en el sentido de que son consideradas como superficies inclinadas por donde transitan ambos coches. Surgen respuestas como la de E13 (Figura 4)

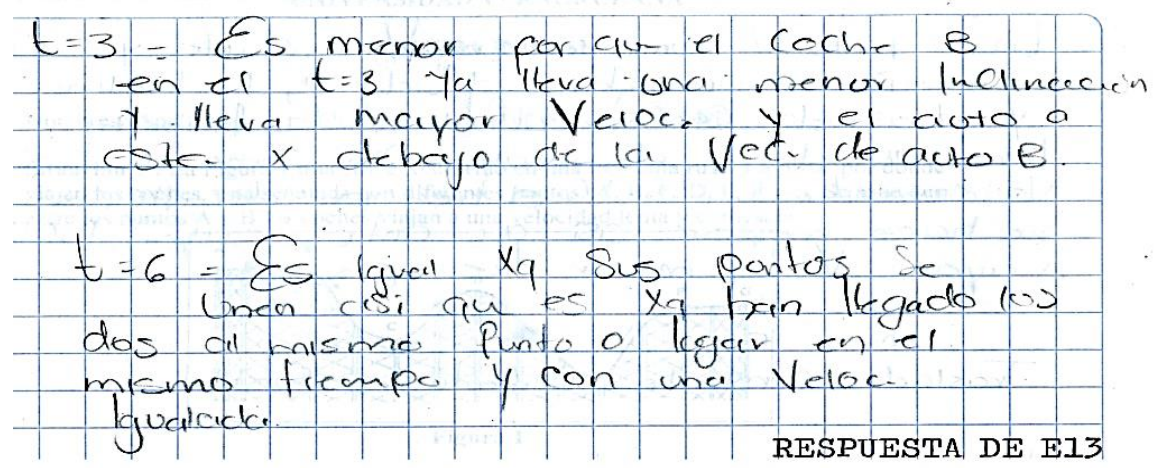

Figura 4. Respuesta del estudiante $\mathrm{E}_{13}$ a la situación 1 
Luego, durante el intercambio de ideas con el profesor, de nuevo interpreta la imagen visual "intersección de ambas rectas" como un "punto de encuentro" de ambos coches.

Profesor ¿Qué coche tiene menor velocidad en $t=3$ ?

$\mathrm{E}_{13} \quad$ El coche A, porque la línea de A está más inclinada y le cuesta más trabajo.

Profesor ¿YY el coche B?

$\mathrm{E}_{13} \quad$ Lleva menos inclinación y mayor velocidad.

Profesor $Y$ en $t=6$, ¿cuál tiene mayor velocidad?

$\mathrm{E}_{13} \quad$ Es igual, porque los dos coches pasan por un mismo punto de la recta, entonces la velocidad es la misma.

Otras: no responde a la pregunta, o la respuesta es totalmente incoherente, como se puestra en la respuesta de $\mathrm{E}_{26}$ (Figura 5).

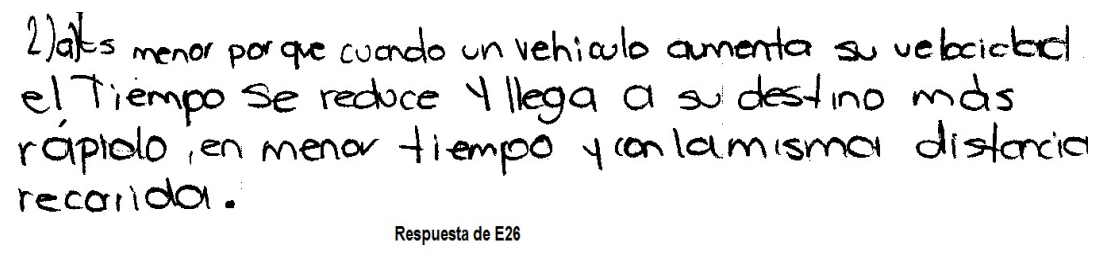

Figura 5. Respuesta del estudiante $\mathrm{E}_{26}$ en la situación 1

\subsection{Situación 2 (tarea de construcción)}

En esta tarea se requiere una reflexión sobre el concepto de distancia en su relación con la velocidad y tiempo, a partir de la comprensión del enunciado y de los valores proporcionados.

Un ciclista sale de casa a dar un paseo, desde las 8 am hasta las 12 del día. Durante la primera hora lleva una velocidad constante de $30 \mathrm{~km} / \mathrm{h}$ y luego descansa una hora. Después del descanso regresa a una velocidad de $15 \mathrm{~km} / \mathrm{h}$.

a) Realiza una tabla de valores donde se represente el tiempo (horas) y la distancia a la que se encuentra de la casa.

b) Realiza una gráfica donde se represente esta situación.

Figura 6. Enunciado de la situación 2

Únicamente el 11\% del grupo (Tabla 2) fue capaz de representar en tabla y gráfica la relación entre ambas variables: distancia (en $\mathrm{Km}$ ) y tiempo (en horas), causado por el efecto combinado de la confusión verbal-tabla/grafica, hallada en el $78 \%$ de las respuestas con la confusión intervalo-punto mostrada en otro $40 \%$. 
Tabla 2. Porcentaje de actuaciones, confusiones y sentidos en la situación 2

\begin{tabular}{llcc}
\hline \multirow{2}{*}{ Actuaciones } & & Apartado a) & Apartado b) \\
\cline { 2 - 4 } & Respuestas Correctas & 11 & 11 \\
\cline { 2 - 4 } $\begin{array}{l}\text { Confusiones recogidas e e } \\
\text { literatura previa }\end{array}$ & Respuestas Incorrectas & 89 & 89 \\
\hline \multirow{3}{*}{$\begin{array}{l}\text { Sentidos con los que se } \\
\text { interpretan las variables verbal-tabla/gráfica }\end{array}$} & $\begin{array}{l}\text { Confusión intervalo-punto } \\
\text { Identifica velocidad como }\end{array}$ & 48 & 78 \\
\cline { 2 - 4 } & Iistancia & 67 & 67 \\
\cline { 2 - 4 } & $\begin{array}{l}\text { Inentifica intervalo como punto } \\
\text { enunciado }\end{array}$ & 37 & 37 \\
\cline { 2 - 4 } & Representa por prototipos & 56 & 56 \\
\hline
\end{tabular}

La interpretación personal del estudiante resultó ser un obstáculo para establecer la relación existente entre las variables explicitadas (la velocidad y el tiempo) con la variable objeto de la pregunta (la distancia). Las lecturas personales más relevantes, no excluyentes entre sí, se pueden clasificar como sigue:

Identifica velocidad como distancia: el estudiante responde con datos de tiempo y velocidad, literalmente tomados del planteamiento de la situación. Ejemplo de esta actuación se halla en la respuesta de $\mathrm{E}_{1}$ (Figura 7).

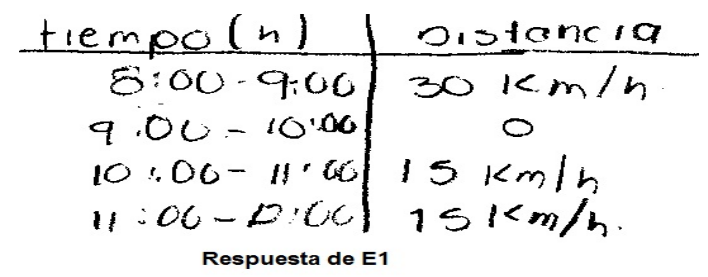

Figura 7. Respuesta del estudiante $\mathrm{E}_{1}$ en la situación 2

Este tipo de respuesta, del $67 \%$ de los estudiantes, puede estar causada por: (i) las experiencias previas con ejemplos sencillos de transferencia de datos desde enunciados verbales, y/o (ii) el desconocimiento de la relación existente entre las variables implicadas en el problema. Para E1 la tarea posee una dificultad que no logra solventar.

Profesor Observa que la indicación es: "realiza una tabla de valores donde se represente el tiempo (en horas) y la distancia a la que se encuentra de la casa".

$\mathrm{E}_{1} \quad$ Es que copié los datos del problema para responder.

Profesor ¿Te preguntan por velocidad o por distancia?

$\mathrm{E}_{1} \mathrm{Si}$, es por la distancia, lo que pasa es que no supe cómo hacerlo, no pude convertir.

Identifica intervalo como punto: además, de no poder establecer la relación existente entre las variables implicadas en el problema, se responde con una tabla que relaciona (Figura 8) valores discretos, a consecuencia de la no comprensión del sentido 
de continuidad de ambas variables (tiempo y distancia). Obsérvese, que el estudiante necesita colocar puntos en el plano cartesiano, tomados literalmente de los datos del problema, para luego unir con segmentos de recta.
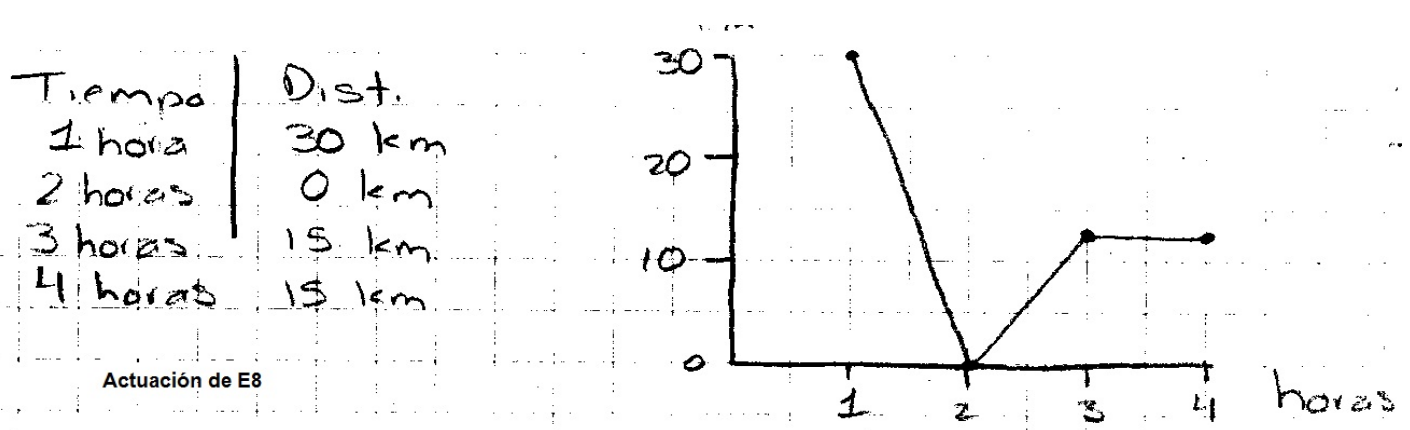

Figura 8. Respuesta del estudiante $\mathrm{E}_{8}$ a la situación 2

Interpretación del enunciado: el estudiante realiza una inadecuada lectura de una frase: "después del descanso regresa a una velocidad de $15 \mathrm{~km} / \mathrm{h}$ " y en consecuencia responde con una tabla de valores siempre crecientes de la distancia (Figura 9).

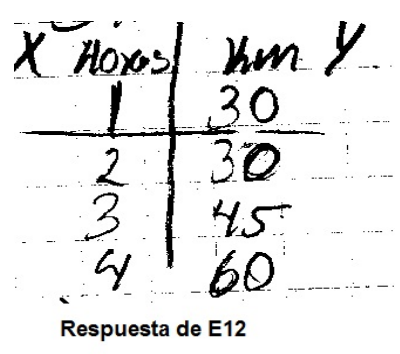

Figura 9. Respuesta del estudiante $\mathrm{E}_{12}$ a la situación 2

Representa por prototipo: se realiza una gráfica (Figura 10) que, lejos de expresar comprensión a la situación planteada, es un prototipo de gráfica (de barras) muy trabajada en la educación preuniversitaria.

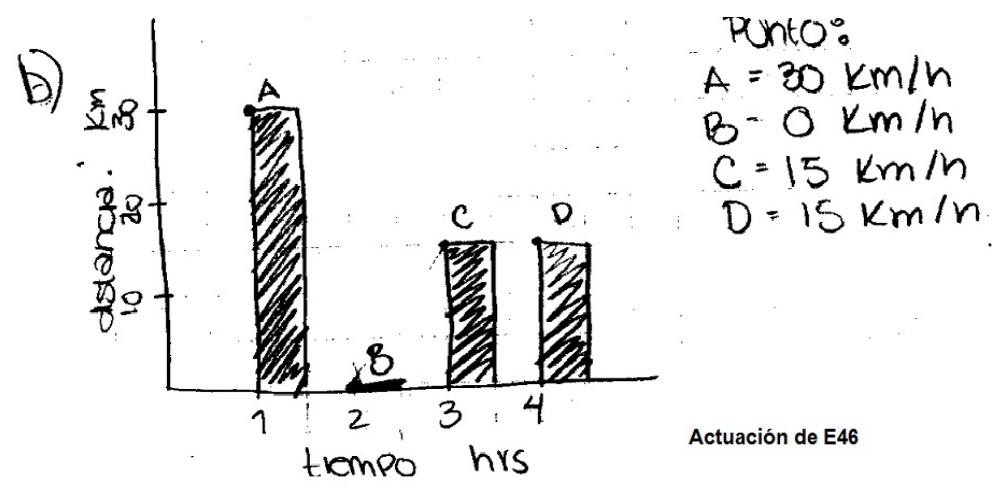

Figura 10. Respuesta del estudiante E46 en la situación 2 


\subsection{Situación 3 (tarea de interpretación y construcción)}

Su solución parte de una interpretación del comportamiento de la velocidad en las diferentes etapas del recorrido, para luego representar sus cambios de manera gráfica.

La figura muestra el recorrido en una montaña rusa. La pista, por donde viajan los coches, está señalada con diferentes puntos: A, B, C, D, E, F y G. Se sabe que entre los puntos A y B los coches viajan a una velocidad lenta y constante.

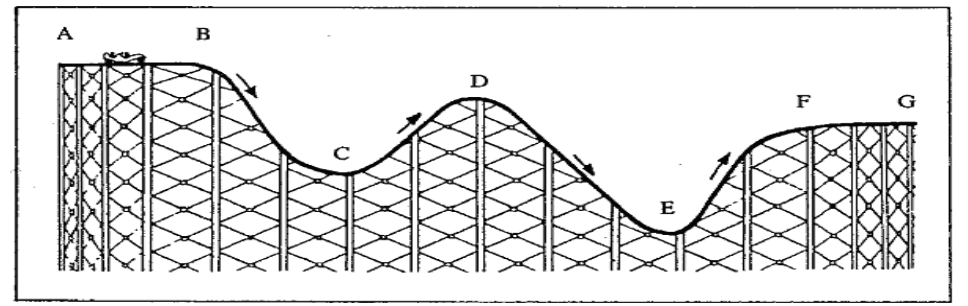

(a) ¿Cómo varía la velocidad del coche durante el recorrido que va desde el punto A hasta el punto G? Explica por escrito, con tus palabras.

(b) Describe tu respuesta anterior mediante una gráfica.

Figura 11. Enunciado de la situación 3

En general, existe adecuada comprensión de la situación; el $92 \%$ de los estudiantes (Tabla 3) logra interpretar la tarea y expone (por escrito) cómo varía la velocidad en función de las características de los diferentes segmentos del recorrido. Sin embargo, sólo el 11\% del grupo es capaz de representar de manera gráfica (respuesta al apartado b) la variación de la velocidad. De este modo, la descripción gráfica no constituye un reflejo del nivel de comprensión mostrado; por el contrario, se halla influenciada (51\% de los casos) por la confusión gráfica-dibujo y por la confusión intervalo-punto ( $30 \%$ de los casos).

Tabla 3. Porcentaje de actuaciones, confusiones y sentidos en la situación 3

\begin{tabular}{llcc}
\hline & & Apartado a) & Apartado b) \\
\cline { 2 - 3 } Actuaciones & Respuestas Correctas & 92 & 11 \\
& Respuestas Incorrectas & 8 & 89 \\
\hline $\begin{array}{l}\text { Confusiones recogidas en } \\
\text { investigaciones previas }\end{array}$ & Confusión gráfico-dibujo & 51 \\
\hline & Confusión intervalo-punto & 30 \\
\hline $\begin{array}{l}\text { Sentidos con los que se } \\
\text { interpretan las variables }\end{array}$ & Interpretación del dibujo & 51 \\
& Identifica altura como velocidad & 54 \\
& $\begin{array}{l}\text { Interpretación incorrecta del } \\
\text { enunciado }\end{array}$ & 24 \\
\hline
\end{tabular}

Tal confusión se produce por las lecturas que se realizan del propio enunciado y/o de la imagen mostrada, así como de las variables involucradas (velocidad y tiempo). Lecturas que se pueden resumir en: 
Interpretación del dibujo: con independencia del nivel de comprensión sobre los cambios de velocidad, muchos estudiantes (51\% del grupo) esbozan una gráfica que es, en esencia, una imagen exacta (Figura 12) de la montaña

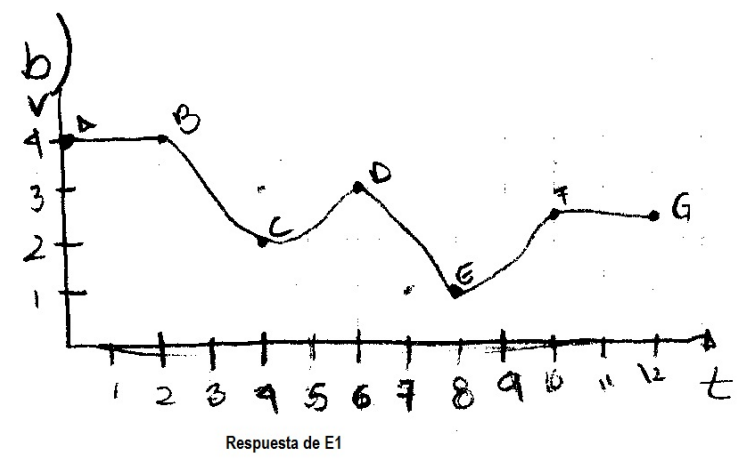

Figura 12. Respuesta del estudiante $\mathrm{E}_{1}$ en la situación 3

El estudiante interpreta de manera adecuada los cambios de velocidad del coche, pero la gráfica es una imagen literal, es decir una representación icónica.

Profesor ¿Cuál es tu respuesta a esta pregunta?

$\mathrm{E}_{20} \quad$ Yo escribi que desde $A$ hasta $B$ la velocidad es lenta y desde $B$ hasta $C$ es rápida.

Profesor ¿Por qué es rápida de $B$ a C?

$\mathrm{E}_{20} \quad$ Porque el coche baja la montaña.

Profesor Pero en tu respuesta gráfica la velocidad no aumenta, al contrario disminuye.

$\mathrm{E}_{20} \quad$ Es que me guie por el dibujo, me confunde cómo va el coche.

Identifica altura como velocidad: se representa la velocidad asociada a la altura del eje vertical en el sentido siguiente: cuando la velocidad baja (disminuye) se asume como que es una "baja velocidad" y en consecuencia debe tener un valor pequeño; por el contrario, cuando la velocidad sube (aumenta) debe tener un valor alto. Es decir, cada segmento del recorrido (Figura 13) se hace corresponder a valores (bajos o altos) de velocidad, que luego se unen por segmentos de recta

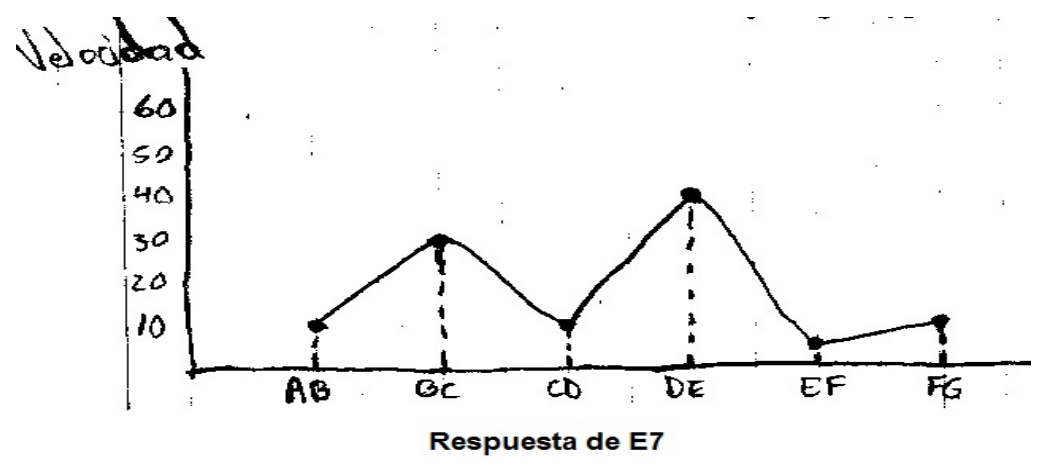

Figura 13. Respuesta del estudiante $\mathrm{E}_{7}$ en la situación 3 
Representación por prototipos: La representación gráfica (24\% del grupo) asocia el comportamiento de la velocidad a un tipo especial de grafica (de barras) que es, en esencia, un modelo conocido (Figura 14) y quizás muy trabajado en el aula. A ello se une la necesidad de situar valores en los ejes para representar el cambio de velocidad

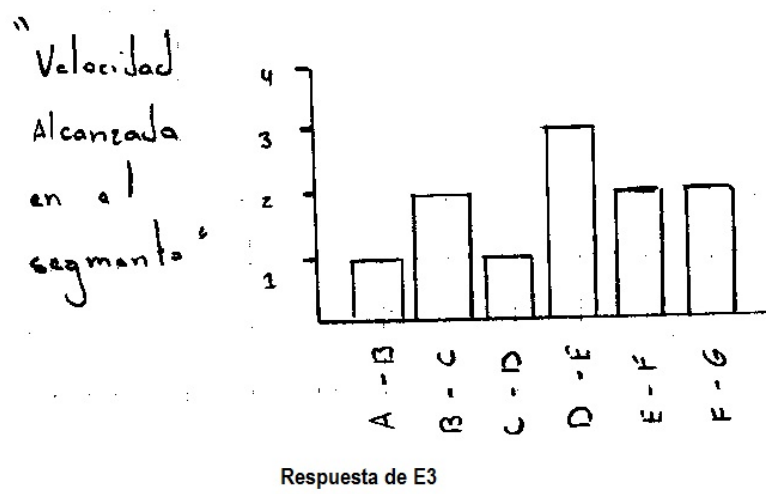

Figura 14. Respuesta del estudiante $\mathrm{E}_{3}$ en la situación 3

Profesor ¿Cómo representas en forma gráfica?

$\mathrm{E}_{3} \quad$ Marqué uno, dos, tres y cuatro como niveles de velocidad [hace referencia a la gráfica] entonces $A B$ es que va en el nivel uno, el tramo $B C$ va en el nivel dos porque tiene mayor velocidad, en el tramo $C D$ disminuye la velocidad, y en $D E$ incrementa el doble porque es el doble de pendiente....

Profesor ¿Por qué una gráfica de barras?

$\mathrm{E}_{3} \quad$ Es que dice que explique cómo varía la velocidad, en el gráfico explico cuánta velocidad lleva el coche en cada segmento.

Profesor ¿Por qué con barras?

$\mathrm{E}_{3} \quad$ Es que con lineas no entendería los niveles de velocidad.

Interpretación incorrecta del enunciado: se representa otro tipo de relación, en muchos casos sin sentido (Figura 15) en el contexto de la situación propuesta.

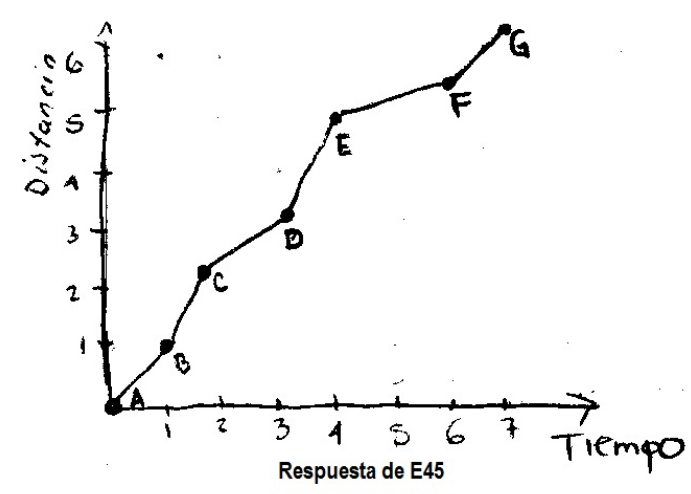

Figura 15. Respuesta del estudiante $\mathrm{E}_{45}$ en la situación 3 
En resumen, las respuestas a las tres situaciones analizadas ponen de manifiesto la existencia de dificultades asociadas, no solo a la interpretación del enunciado de la pregunta, sino también a la comprensión del contexto en que se propone la tarea. Las lecturas personales del estudiante poseen un patrón común: el estudiante intenta resolver las tareas con la información que le es directamente accesible en la lectura de las imágenes, las descripciones verbales y los datos proporcionados.

\section{Conclusiones}

El estudio pudo corroborar la existencia de errores en tareas de interpretación y/o construcción de gráficas en situaciones contextualizadas. Muestra la existencia de errores en las respuestas del estudiante, causados por la forma en que los estudiantes entienden y comunican su significado de "velocidad". En las actuaciones se observan cuatro confusiones recogidas en la bibliografía (Leinhardt et al., 1990): (i) la confusión entre la pendiente de una recta y su altura, (ii) la consideración de una gráfica de una situación contextualizada como un dibujo, (iii) la confusión entre un intervalo y un punto, (iv) la confusión entre el enunciado verbal de una situación contextualizada y las representaciones en forma de tabla y/o gráfica (Ortega \& Pecharromán, 2014), y (v) el uso de gráficos no apropiados para representaciones funcionales de velocidad (Dolores et al., 2009).

Además, desde un punto de vista semántico y considerando el sentido que los estudiantes comunican a través de sus explicaciones, los aspectos más relevantes que hemos detectado son:

1. La incomprensión del significado de la pendiente de la recta. No se reconoce, en la gráfica de una situación contextualizada, la velocidad como la pendiente de la recta; por el contrario, se identifica la pendiente a la altura vertical de la recta en la gráfica. Todos los estudiantes, de acuerdo con las entrevistas realizadas, poseen un conocimiento algorítmico para calcular velocidad, dados distancia y tiempo, que predomina sobre el conocimiento conceptual (geométrico y funcional).

2. La incomprensión de la relación entre variables mostradas en la gráfica. La situación contextualizada se analiza atendiendo solamente a una lectura literal de las magnitudes involucradas, sin establecer la relación manifiesta entre ellas. Este resultado es coincidente con la idea expuesta por Deulofeu (1995) sobre la tendencia a discretizar una situación, considerando únicamente los puntos relevantes de la gráfica y restringiendo la dependencia funcional entre variables a las coordenadas de tales puntos.

3. La interpretación icónica de situaciones gráficas contextualizadas. Muchos estudiantes realizan una interpretación icónica de los cambios de la velocidad de un coche que sube y baja una montaña rusa; para estos estudiantes la gráfica que debe representar los cambios de velocidad es, en esencia, una representación (una imagen) de la montaña rusa. Este resultado constituye uno de los errores conceptuales en el sentido de Leinhardt et ál. (1990): el estudiante interpreta la gráfica de una situación como una imagen literal de esa situación, es decir una representación icónica.

Lo hallado en esta investigación pone en evidencia la existencia de un problema relacionado con la ausencia de significados en los estudiantes sobre los conceptos de velocidad, distancia recorrida y pendiente de recta, cuando se enfrentan a tareas sobre 
situaciones contextualizadas de manera verbal y/o gráfica. Un concepto como velocidad, y su relación con las nociones de distancia y tiempo, no ha sido construido por los estudiantes de manera satisfactoria. Resultados, que podrían deberse a un tipo de enseñanza con especial énfasis en los aspectos manipulativos y que no coadyuva al proceso de comprensión del estudiante.

Se pudo corroborar que, en efecto, el significado de función y de relación entre variables, que expresan los estudiantes, parece ser insuficiente para acceder a las complejas estructuras y relaciones que exige el estudio de las funciones, y en particular sus distintos sistemas de representación como el lenguaje gráfico.

En resumen, las actuaciones mostradas ponen en evidencia la existencia de un problema relacionado con el nivel de conocimiento con el que los estudiantes ingresan a la universidad. Se constata que conceptos fundamentales como velocidad y pendiente de una recta no han sido construidos de manera apropiada, lo cual constituye un obstáculo para abordar analíticamente el estudio de la matemática universitaria.

\section{Referencias}

Azcárate, C. \& Deulofeu, J. (Eds.) (1990). Funciones y gráficas. Madrid: Editorial Síntesis.

Bagni, G.T. (2004). Una experiencia didáctica sobre funciones en la escuela secundaria. RELIME, Revista Latinoamericana de Investigación en Matemática Educativa 7(1), 5-23.

Castro, E., \& Castro, E. (1997). Representaciones y modelización. En L. Rico (Ed.), La educación matemática en la enseñanza secundaria (pp. 95-124). Barcelona: Horsori.

Cohen, L., Manion, L., \& Morrison, K. (2011). Research methods in education. Londres: Routledge.

Cuesta, A. (2007). El proceso de aprendizaje de los conceptos de función y extremo de una función en estudiantes de economía: análisis de una innovación educativa. (Tesis doctoral no publicada). Universidad Autónoma de Barcelona, Bellaterra, España.

Davis, R., Young, S., \& McLoughlin, P. (1982). The roles of "understanding” in the learning of mathematics. Urbana/Champaign Curriculum Laboratory: University of Illinois.

Deulofeu, J. (1993). Els gràfics cartesians de funcions: un estudi de les concepcions dels alumnes centrat en el significat del gràfic. (Tesis doctoral no publicada). Universidad Autónoma de Barcelona, Bellatera, España.

Deulofeu, J. (1995). Concepciones de los alumnos de secundaria sobre distintas gráficas de funciones. UNO. Revista de Didáctica de las Matemáticas, 4, 6-16.

Dolores, C. (2004). Acerca del análisis de funciones a través de sus gráficas: concepciones alternativas de estudiantes de bachillerato. RELIME. Revista Latinoamericana de Investigación en Matemática Educativa, 7(3), 195-218.

Dolores, C., Bello, G.A., \& Millán, D.F.A. (2002). Concepciones alternativas sobre las gráficas cartesianas del movimiento: el caso de la velocidad y la trayectoria. RELIME. Revista Latinoamericana de Investigación en Matemática Educativa, 5(3), 225-250.

Dolores, C., Chi, A.G., Canul, E.R., Cantú, C.A., \& Pastor, C.G. (2009). De las descripciones verbales a las representaciones gráficas. El caso de la rapidez de la variación en la enseñanza de la matemática. Unión, 18, 41 - 57.

Duval, R. (1988). Gráficas y ecuaciones: la articulación de dos registros. En R. Cambray, E. Sánchez \& G. Zubieta (Comp.), Antología en Educación Matemática, Material de apoyo para el seminario de educación matemática I (pp. 125-141). México: CINVESTAV-IPN. 
Duval, R. (1999). Semiosis y pensamiento humano. Registros semióticos de aprendizajes intelectuales. Cali, Colombia: Universidad del Valle.

Fabra, M., \& Deulofeu, J. (2000). Construcción de gráficos de funciones: Continuidad y prototipos. RELIME, Revista Latinoamericana de Investigación en Matemática Educativa $3(2), 207-230$.

Frege, G. (2013). Sobre sentido y referencia. En L.M. Valdés (Ed.), Ensayos de semántica y filosofía de la lógica. (pp. 84-111) Madrid: Tecnos.

Goldin, G. (1998). Representations and the psychology of mathematics education: Part II. Journal of Mathematical Behaviour 17(2), 137-165.

Hitt, F. (2001) El papel de los esquemas, las conexiones y las representaciones internas y externas dentro de un Proyecto de Investigación en Educación Matemática. En P. Gómez \& L. Rico (Eds.), Iniciación a la investigación en didáctica de la matemática. Homenaje al profesor Mauricio Castro (pp. 165-177). Granada: Universidad de Granada.

Janvier, C. (1987). Representations and understanding: The notion of function as an example. En C. Janvier (Ed.) Problems of representation in the teaching and learning of mathematics (pp. 67-71). NJ: Lawrence Erlbaum.

Jiang, C. (2009). Word problems on speed: students' strategies and errors. En K.Y. Wong (Ed.), Mathematics Education: The Singapore Journey (pp. 244-262). Singapore; Word Scientific.

Jiang, C. (2013). Errors in solving word problems about speed: A case in Singapore and Mainland China. New Waves-Educational Research \& Development, 16(1), 56-75.

Jiang, C., \& Chua, B. L. (2010). Strategies for solving three fraction-related word problems on speed: A comparative study between Chinese and Singaporean students. International Journal of Science and Mathematics Education, 8(1), 73-96.

Jiang, C., Hwang, S., \& Cai, J. (2014). Chinese and Singaporean sixth-grade students' strategies for solving problems about speed. Educational Studies in Mathematics, 87(1), 27-50.

Leinhardt, G., Zaslavsky, O., \& Stein, M. (1990). Functions, graphs, and graphing: Tasks, learning and teaching. Review of Educational Research 60(1), 1-64.

Martí, E., \& Pozo, J.I. (2000). Más allá de las representaciones mentales: la adquisición de los sistemas externos de representación. Infancia y aprendizaje, 23(90), 11-30.

Ortega, T., \& Pecharromán, C. (2014). Errores en el aprendizaje de las propiedades globales de las funciones. Revista de Investigación en Educación 12(2), 209-221.

Rico, L. (2009). Sobre las nociones de representación y comprensión en la investigación en educación matemática. PNA 4(1), 1-14.

Rico, L. (2012). Aproximación a la investigación en Didáctica de la Matemática. Avances en Investigación en Educación Matemática 1, 39-63.

Rico, L. \& Fernández-Cano, A. (2013). Análisis didáctico y metodología de investigación. En L. Rico, J. L. Lupiáñez, \& M. Molina (Eds.), Análisis didáctico en educación matemática (pp. 1-22). Granada: Comares.

Shell Centre for Mathematical Education (1990). El lenguaje de funciones y gráficas. Ministerio de Educación y Cultura. Universidad del País Vasco.

Zhou, Z., Peverly, S. T., Boehm, A. E., \& Lin, C. (2000). American and Chinese children's understanding of distance, time, and speed interrelations. Cognitive Development, 15(2), 215-240. 
Zhou, Z., Peverly, S. T., \& Lin, C. (2004). Cross-and within-cultural variations in children's understanding of distance, time, and speed interrelationships: A follow-up study. The Journal of genetic psychology, 165(1), 5-27.

\section{Referencias de los autores}

Abraham Cuesta Borges , Universidad Veracruzana (México). acuesta@uv.mx Juana Elisa Escalante Vega, Universidad Veracruzana (México). jescalante@uv.mx Juan Francisco Ruiz Hidalgo, Universidad de Granada (España).jfruiz@ugr.es

\section{Anexo Tareas del cuestionario no analizadas.}

Situación 4. Manuel está enfermo, su madre le toma la temperatura en varias ocasiones y obtiene las siguientes mediciones:

- A las 10 horas la temperatura fue de $370 \mathrm{C}$ y dos horas después tenía $390 \mathrm{C}$,

- A las 14: 00 horas tenía $380 \mathrm{C}$ y continuó igual hasta las 16: 00 horas,

- $\quad$ A las 18 horas tenía $360 \mathrm{C}$ y a las 20 horas había aumentado en $20 \mathrm{C}$.

(a) Construye una tabla de valores con las mediciones obtenidas de la temperatura.

(b) Representa esta situación en una gráfica.

(c) ¿En qué momento la temperatura debió ser de $380 \mathrm{C}$ ?

(d) ¿En qué intervalos de tiempo Manuel se mantuvo estable?

Situación 5: Una persona, en una bicicleta, realiza la siguiente excursión:

1ra. Fase: Va por un terreno llano.

2da. Fase: Sube una montaña.

3ra. Fase: Baja la montaña.

4ta. Fase: Va, de nuevo, por un terreno llano.

(a) Dibuja la gráfica: "Cambio de velocidad con respecto al tiempo". Explica la gráfica.

(b) ¿La velocidad es una función que depende del tiempo? Explica

(c) ¿Puedes decir algo sobre crecimiento y/o decrecimiento en esta situación? 


\title{
Speed. Meanings expressed by college students from graphs
}

\author{
Abraham Cuesta Borges, Universidad Veracruzana (México). \\ Juana Elisa Escalante Vega, Universidad Veracruzana (México). \\ Juan Francisco Ruiz Hidalgo, Universidad de Granada (España).
}

Concerned by the students' results obtained in Mathematics in the degree of Economics, their teachers proposed a levelling course of Calculus. Functions and its graphical representations were part of the main topics within the course, due to the influence of this topic on the comprehension of different economic theories. Within the course, by means of a sequence of tasks, students are led through the function concept and its global properties.

Previously, the teachers decided to apply a diagnostic evaluation, and used the results for this research. We formulated five questions about functions, especially focused on their graphical representations and on interpreting their global properties. The goal is to detect the meanings that students express about each variable from the tasks. More concretely, we focus the attention on the speed concept. The issue is to determinate whether the students interpret and construct graphs in contexts, as well as if they identify their global properties.

We detected difficulties related to two known confusions (Janvier, 1987): graphas-picture and verbal-graphical confusions. Moreover, students did not interpret functional dependence properly and also they expressed misunderstandings of the meaning of slope.

In addition, we detected lack of meaning for the speed concept in the students' answers, concreted in three problems of sense: firstly, lack of meaning of slope; secondly, lack of meaning of the relation among variables; and finally, understanding graphs as functions.

The results show that grasping meaning of function and relation among variables expressed by students are not enough to understand the complex structures and the different graphical representations appearing in the graphical language. 